\title{
Biolixiviation des métaux lourds et stabilisation des boues municipales: effet de la forme du souffre élémentaire utilisé comme substrat
}

\section{Heavy metal bioleaching and stabilization of municipal sludges : effect of the form of elemental sulfur used as substrate}

\author{
H. Benmoussa, R. D. Tyagi et P. G.C. Campbell
}

Volume 7, numéro 3, 1994

URI : https://id.erudit.org/iderudit/705199ar

DOI : https://doi.org/10.7202/705199ar

Aller au sommaire du numéro

\section{Éditeur(s)}

Université du Québec - INRS-Eau, Terre et Environnement (INRS-ETE)

ISSN

0992-7158 (imprimé)

1718-8598 (numérique)

Découvrir la revue

Citer cet article

Benmoussa, H., Tyagi, R. D. \& Campbell, P. G. (1994). Biolixiviation des métaux lourds et stabilisation des boues municipales: effet de la forme du souffre élémentaire utilisé comme substrat. Revue des sciences de l'eau / Journal of Water Science, 7(3), 235-250. https://doi.org/10.7202/705199ar

\section{Résumé de l'article}

La présence de concentrations élevées en métaux lourds retrouvés dans les boues de stations d'épuration est un facteur important limitant ainsi leurs différents modes de dispositions (rejet en mer, enfouissement, incinération, ou même recyclage comme fertilisant agricole ou forestier) par crainte de dégâts considérables qui pourraient être causés à l'environnement. L'objectif de ce travail consistait à mettre au point un procédé qui permettrait conjointement la stabilisation des boues municipales et l'enlèvement des métaux lourds qui y sont associés. L'approche poursuivie dans cette étude était de vérifier si ce procédé microbien, conçu pour enlever les métaux lourds associés aux boues municipales, pourrait éventuellement remplacer les procédés conventionnels de stabilisation des boues municipales, ce qui réduirait considérablement les temps et coûts de traitement. Ce procédé microbien consiste à utiliser des souches de thiobacilles qui oxydent le soufre élémentaire, produisant ainsi de l'acide sulfurique, et par la même occasion une forte baisse de $\mathrm{pH}$ (1.5). Les résultats obtenus en cultures discontinues, en bioréacteurs, avec des boues primaires provenant de la station d'épuration de la Communauté Urbaine de Québec (C.U.Q.- Est) montrent que cette chute de pH entraîne une solubilisation importante des métaux (Cr: $56 \%$, Cu: 97 \%, Fe: 30 à $40 \%$, Pb: $69 \%$, Zn: $98 \%$ ), du phosphore (52\%), ainsi qu'une réduction appréciable des matières volatiles en suspension ( 40 à $50 \%$ ), et ce, après seulement 7 jours de traitement. Le soufre nécessaire à la biolixiviation- stabilisation est introduit, dans le cas de notre étude, sous forme de granules ou de blocs. Ce choix de la forme de soufre influe beaucoup sur la qualité de la boue produite, ainsi que sur son pouvoir acidophile après neutralisation. Le soufre en blocs s'avère plus efficace et aussi préférable au soufre en granules quant au pouvoir acidophile, après neutralisation, de la boue produite.
Ce document est protégé par la loi sur le droit d'auteur. L’utilisation des services d'Érudit (y compris la reproduction) est assujettie à sa politique d'utilisation que vous pouvez consulter en ligne.

https://apropos.erudit.org/fr/usagers/politique-dutilisation/ 


\title{
Biolixiviation des métaux lourds et stabilisation des boues municipales: effet de la forme du soufre élémentaire utilisé comme substrat
}

\author{
Heavy metal bioleaching and stabilization \\ of municipal sludges : effect of the form \\ of elemental sulfur used as substrate
}

\section{H. BENMOUSSA ${ }^{1}, 2$, R.D. TYAGI ${ }^{\text {* }}$ et P.G.C. CAMPBELL1}

Reçu le 6 janvier 1994, accepté le 27 mai 1994**.

\section{SUMMARY}

Given the potential geochemical mobility and recognized toxicity of heavy metals, their presence at high concentrations in sewage sludges imposes serious limitations on various sludge disposal practices (ocean disposal, landfill, incineration, or use as a fertilizer in agriculture or forestry). The objective of this work was to develop a process that would permit the simultaneous stabilization of sewage siudges and the removal of heavy metals associated with them. The approach followed was to verify if this microbial leaching procedure could eventually replace conventional sludge stabilization processes and hence considerably reduce the time and cost of treatment. The microbial process consists of using thiobacillus strains which, in the presence of air, oxidize elemental sulfur to sulfuric acid, thus reducing the $\mathrm{pH}$ to very acidic levels (pH 1.5). This biological oxidation of elemental sulfur is brought about by two groups of sulfur-oxidizing bacteria, the less-acidophilic and the acidophilic thiobacilli. The initial acid production and pH reduction is due to the less-acidophilic bacteria (Thiobacillus thioparus) which lower the pH to about 4.0. This is followed by the growth of acidophilic bacteria (Thiobacillus thiooxidans) and further $\mathrm{pH}$ reduction.

Batch culture experiments were carried out in $30 \mathrm{~L}$ and $8 \mathrm{~L}$ reactors with primary sludges obtained from the Quebec urban community's wastewater treatment centre. Elemental sulfur and inoculum were added at the beginning of each experiment. The inoculum was prepared by adding $1 \%$ tyndalized sulfur powder to fresh secondary sludge and incubating for 8 days (final pH 1.5 to 2.0). A small portion $(5 \%)$ of this acidified sludge was then used as an inoculum for another batch of fresh studge and this process was repeated several times until an acclimatized inoculum was obtained which could oxidize elemental sulfur rapidly, without an appreciable lag phase. The elemental sulfur necessary as substrate for the simultaneous bioleaching and sludge stabilization was introduced in the form of granules ( 2.4 to $4 \mathrm{~mm}$ diameter) or blocks ( $25 \mathrm{~mm}$ diameter).

1. Institut national de la recherche scientifique (INRS-Eau), Université du Québec, 2700 rue Einstein, Sainte-Foy, Québec, Canada GiV 4 C7.

2. Université de Batna, Institut d'Hygiène et Sécurité, 05000 Batna, Algérie.

* Auteur correspondant.

* Les commentaires seront reçus jusqu'au 31 mars 1995. 
Sludge $\mathrm{pH}$ and ORP were measured at 24 hour intervals and all other measurements were carried out at $\mathbf{4 8}$ hour intervals. The results demonstrate that the addition of elemental sulfur and inoculum resulted in a considerable lowering of the sludge pH during the incubation period. Such pH lowering was not observed in cultures to which sulfur and inoculum were not added. This lowering of $\mathrm{pH}$ (2.1) was related to the quantity of substrate (sulfur) and inoculum present in the medium. An increase in the medium ORP (from -50 to about $500 \mathrm{mV}$ ) was also observed. Acidification of the medium along with the elevated ORP levels resulted in the solubilization of metals initially present in the sludge. This $\mathrm{pH}$ reduction, after only 7 days of treatment, effected an important metal solubilization (Cr: $56 \%$, Cu: $97 \%$, Fe: 30-40\%, Pb: $69 \%, \mathrm{Zn}: 98 \%$ ), as well as an appreciable reduction in phosphorus $(52 \%)$ and in the volatile suspended solids concentration (40-50\%). In addition, the sludge which initially had a highly repulsive odour was rendered odourless.

In a previous study we had shown that for an optimum rate of acidification of the sludge a minimum concentration of elemental sulfur $(2 \mathrm{~g} / \mathrm{L})$ was necessary, even though only $40 \%$ of this sulfur was oxidized. In the present experiment the physical form of the sulfur was shown to influence both the quality of sludge produced and its acid-generating capacity after neutralization. Sulfur in the form of blocks was more efficient than granules in that the elemental sulfur could be readily separated from the sludge at the end of the treatment, yielding a sludge after neutralization that had a low acid-generating capacity. In the case of granules, the unused sulfur was broken down into a fine powder during the course of the bioleaching experiment and could not be separated from the leached sludge - even after neutralization, the leached sludge exhibited a high acidgenerating capacity, which would limit its use as a fertilizer on agricultural land.

Key-words : heavy metals, sewage, elemental sulfur, bioleaching, sludge stabilization.

\section{RÉSUMÉ}

La présence de concentrations élevées en métaux lourds retrouvés dans les boues de stations d'épuration est un facteur important limitant ainsi leurs différents modes de dispositions (rejet en mer, enfouissement, incinération, ou même recyclage comme fertilisant agricole ou forestier) par crainte de dégâts considérables qui pourraient être causés à l'environnement. L'objectif de ce travail consistait à mettre au point un procédé qui permettrait conjointement la stabilisation des boues municipales et l'enlèvement des métaux lourds qui y sont associés. L'approche poursuivie dans cette étude était de vérifier si ce procédé microbien, conçu pour enlever les métaux lourds associés aux boues municipales, pourrait éventuellement remplacer les procédés conventionnels de stabilisation des boues municipales, ce qui réduirait considérablement les temps et coûts de traitement. Ce procédé microbien consiste à utiliser des souches de thiobacilles qui oxydent le soufre élémentaire, produisant ainsi de l'acide sulfurique, et par la même occasion une forte baisse de $\mathrm{pH}$ (1.5). Les résultats obtenus en cultures discontinues, en bioréacteurs, avec des boues primaires provenant de la station d'épuration de la conmunauté urbaine de Québec (C.U.Q.- Est) montrent que cette chute de pH entraîne une solubilisation importante des métaux ( $\mathrm{Cr}: 56 \%, \mathrm{Cu}: 97 \%, \mathrm{Fe}$ : 30 à $40 \%, \mathrm{~Pb}: 69 \%, \mathrm{Zn}: 98 \%$ ), du phosphore (52\%), ainsi qu'une réduction appréciable des matières volatiles en suspension ( 40 à $50 \%$ ), et ce, après seulement 7 jours de traitement. Le soufre nécessaire à la biolixiviationstabilisation est introduit, dans le cas de notre étude, sous forme de granules ou de blocs. Ce choix de la forme de soufre influe beaucoup sur la qualité de la boue produite, ainsi que sur son pouvoir acidophile aprìs neutralisation. Le soufre en blocs s'avère plus efficace et aussi préférable au soufre en granules quant au pouvoir acidophile, après neutralisation, de la boue produrte.

Mots clés : métaux lourds, boues d'épuration, soufre élémentaire, biolixiviation, stabilisation des boues. 


\section{INTRODUCTION}

L'épandage agricole des boues d'épuration est un mode d'utilisation indispensable pour faire face à l'augmentation importante des quantités de boues résiduaires générées par le traitement des eaux usées municipales (BRUCE et DAVIS, 1989 ; U.S. EPA, 1990 ; WEBBER, 1988). De plus, cette pra tique permet de tirer profit des propriétés des boues pour la préparation et la fertilisation des sols (DAVIS, 1987 ; U.S. EPA, 1979). Toutefois, la non stabilisa tion des boues, ainsi que les teneurs élevées en métaux lourds qu'elles contiennent, et qui se situent entre 0,5 et $2 \%$ sur une base de poids sec pouvant même atteindre $6 \%$ dans des cas extrêmes (LESTER et al., 1983), posent de sérieuses contraintes face à cette pratique (BRUCE et DAVIS, 1989; CHANEY, 1990 ; MININNI et SANTORI, 1987). L'assimilation de certains métaux par les plantes et leur accumulation subséquente dans la chaine alimentaire (TYLER et al., 1989) sont des problèmes importants qui ont stimulé la recherche pour le développement de techniques d'enlèvement des métaux lourds applicables aux boues municipales et industrielles.

Si certains procédés chimiques donnent de bons rendements d'extraction, notamment le traitement à l'acide (WOZNIAK et HUANG, 1982), les coûts élevés d'opération rendent ces techniques peu attrayantes. Plus récemment, quelques procédés biologiques de lixiviation des métaux ont été étudiés (BLAIS et al., 1992a ; COUILLARD et MERCIER, 1991a, b; TYAGI et al., 1990 ; WONG et HENRY, 1983, 1988). Les caractéristiques principales recherchées lors de la mise au point d'une telle biotechnologie sont les suivantes: court temps de résidence ; rendement adéquat d'enlèvement des métaux; utilisation d'un substrat peu coûteux pour la croissance bactérienne. Le procédé doit être fonctionnel à la température ambiante ainsi qu'au $\mathrm{pH}$ initial de la boue, et surtout utilisable avec divers types de boues. De plus, l'opération doit être peu onéreuse et facilement réalisable. Le présent procédé, qui utilise des souches du genre thiobacilles retrouvées naturellement dans les boues (JAIN et TYAGI, 1992 ; BLAIS et al., 1992a), semble satisfaire à ces exigences. La solubilisation des métaux s'effectue par l'acidification importante des boues, résultant de l'oxydation biologique du soufre élémentaire (substrat) en acide sulfurique (équation 1). Ce phénomène biologique est réalisé par deux genres de thiobacilles (peu-acidophiles et acidophiles). L'acidification initiale des boues s'effectue lors de la croissance des populations indigènes de thiobacilles peuacidophiles (Thibacillus thioparus) qui abaissent le $\mathrm{pH}$ aux environs de 4,0; les thiobacilles acidophiles (Thiobacillus thiooxidans) provenant de l'ajout de l'inoculum se développent ensuite, en provoquant une réduction importante du $\mathrm{pH}$ de la boue (BLAIS et al., 1992a). Ces bactéries peuvent également concourir à la solubilisation des métaux en tirant leur énergie de l'oxydation directe des sulfures de métaux en sulfates solubles (HUTCHINS et al., 1986) selon l'équation 2.

$$
\begin{gathered}
2 \mathrm{~S}^{\circ}+3 \mathrm{O}_{2}+2 \mathrm{H}_{2} \mathrm{O} \rightarrow 2 \mathrm{H}_{2} \mathrm{SO}_{4} \\
\mathrm{MS}+2 \mathrm{O}_{2} \rightarrow \mathrm{MSO}_{4}
\end{gathered}
$$

où MS représente le sulfure du métal $M$. 
Par ailleurs, la stabilisation ou " digestion" des boues par voie aérobie consiste, par une aération prolongée de ces demières, à provoquer le développement de micro-organismes aérobies, jusqu'à dépasser la période de synthèse des cellules et réaliser leur propre auto-oxydation. Ceci conduit à une réduction finale du poids de la boue, suite à une réduction de matière volatile; dans le cas d'une parfaite stabilisation, cette réduction est de 30 à $35 \%$ (DEGRÉMONT, 1989).

L'ajout d'un procédé d'extraction des métaux en aval de la stabilisation conventionnelle des boues serait fortement recommandable d'un point de vue écologique, mais il hausserąit nécessairement les coûts assignés aux traitements des eaux usées. Ainsi, la destruction efficace des bactéries indicatrices de pathogénécité durant la lixiviation ayant été démontrée (BLAIS et al., 1992b), la mise au point d'un procédé intégré, permettant conjointement l'enlèvement des métaux lourds et la stabilisation des boues, semble donc prometteuse. Une telle option réduirait le temps de rétention et les coûts d'opération, par comparaison aux procédés conventionnels de stabilisation des boues d'épuration. De plus, les boues ainsi traitées répondraient aux critères exigés pour l'épandage agricole (stabilisation et enlèvement des métaux), et contribueraient donc à valoriser cette biomasse considérée le plus souvent comme un déchet.

Nos recherches visent donc le développement, à l'échelie de laboratoire, d'un procédé de biolixiviation, utilisant le soufre élémentaire comme substrat, permettant dans une même étape, l'enlèvement des métaux toxiques et une stabilisation adéquate des boues d'épuration municipales, ainsi que la récupération et la réutilisation du soufre non oxydé.

Enfin, pour que les boues ainsi traitées puissent être valorisées en agriculture, il est impératif de les séparer, entièrement, après lixiviation, du soufre résiduel non oxydé. Cette séparation sera, selon la forme de soufre utilisé et son mode de fixation, plus ou moins facile à réaliser. L'objectif de cette étude était donc de trouver le meilleur moyen de fixer le soufre élémentaire, et ce, afin de pouvoir récupérer entièrement tout le soufre qui n'avait pas été oxydé lors de la lixiviation-stabilisation. Pour cela, le soufre était utilisé sous forme de granules de 2.4 à $4 \mathrm{~mm}$ de diamètre, ou de blocs de $25 \mathrm{~mm}$ de diamètre.

\section{MATÉRIEL ET MÉTHODE}

\section{Échantillonnage des boues}

Les boues utilisées pour la réalisation de ces essais, et dont les concentrations en métaux lourds sont présentées au tableau 1 , provenaient de l'épaississeur de la station d'épuration de la communauté urbaine de QuébecEst. Etant trop épaisses, ces boues ont été diluées environ 3 fois avant leur utilisation, et ce, afin d'obtenir des concentrations en solides totaux entre 20 et 
$25 \mathrm{~g} / \mathrm{l}$. Les essais ont été effectués en mode discontinu, dans deux types de réacteurs, selon que le soufre était introduit sous forme de granules ou de blocs.

Tableau 1 Concentrations en métaux des boues utilisées lors des essais de biolixiviation-stabilisation (poids sec).

Table 1 Concentration of metals from sludges used during the bioleachingstabilization experiments (dry wt. basis).

\begin{tabular}{|ccccccc|}
\hline [Métaux] & $\mathrm{Cr}$ & $\mathrm{Clu}$ & $\mathrm{Fe}$ & $\mathrm{Pb}$ & $\mathrm{Zn}$ & $\mathrm{P}$ \\
\hline $\mathrm{mg} / \mathrm{kg} \mathrm{m} . \mathrm{s}$ & 85 & 225 & 7500 & 60 & 1480 & 4890 \\
\hline
\end{tabular}

\section{Soufre élémentaire utilisé}

Les granules de soufre utilisé sont préparés par précipitation de soufre fondu dans de l'eau tiède, selon la technique de LAISHLEY et al. (1986). La granulométrie du soufre utilisé est comprise entre 2.4 et $4 \mathrm{~mm}$. Cette forme de soufre est utilisée dans les réacteurs de 30 litres type RPA (réacteur parfaitement agité).

Quant au soufre en blocs qui a été utilisé en bioréacteur de type colonne, il a été fondu à 120 degrés Celcius, coulé dans des moules sphériques en plastique de $25 \mathrm{~mm}$ de diamètre, et refroidi à température ambiante du laboratoire.

\section{Microorganismes (inoculum)}

L'inoculum utilisé pour les premiers essais de lixiviation bactérienne des métaux est préparé à partir de $150 \mathrm{~mL}$ de boues secondaires, placées dans un Erlenmeyer de $500 \mathrm{~mL}$ et additionnées de $1 \%$ de soufre en poudre tyndalisée. Cette boue est incubée pendant 8 jours dans un agitateur giratoire (New Brunswick Scientific Co., modèle 26) et agitée à $200 \mathrm{tr} / \mathrm{min}$, ce qui permet l'oxydation du soufre élémentaire et l'acidification du milieu jusqu'à un $\mathrm{pH}$ de 1.5 à 2.0 . Un volume de $150 \mathrm{~mL}$ de boues fraîches contenant $1 \%$ de soufre est ensuite ensemencé avec $5 \%$ de la boue précédemment acidifiée, et incubé pendant une autre période de 8 jours. Cette étape est répétée une autre fois, dans les mêmes conditions, afin de produire un inoculum superactif (bien acclimaté) pouvant oxyder très rapidement le soufre élémentaire. Les boues ainsi obtenues sont utilisées comme inoculum. Pour les essais subséquents, l'inoculum provenait des boues précédemment lixiviées en bioréacteurs.

\section{Description des bioréacteurs utilisés}

Selon que le soufre est sous forme de granules ou de blocs, deux types de bioréacteurs sont utilisés : bioréacteurs de type RPA (R1) et (R2) ou bioréacteur de type colonne (R3), avec recirculation des boues (Schéma 1). 


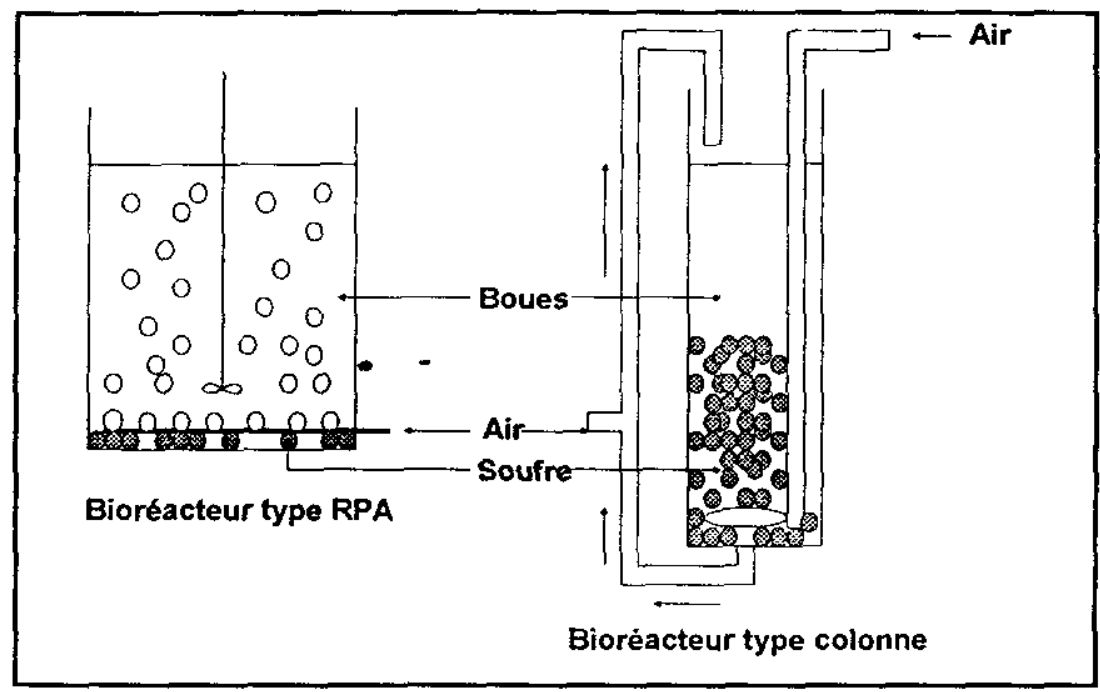

Schéma 1 Bioréacteurs utilisés lors de la biolixiviation-stabilisation.

Bioreactors used during bioleaching-stabilization.

\section{Bioréacteurs de type RPA (R1 et R2)}

Ces bioréacteurs reçoivent chacun 30 litres de boues, avec $20 \%$ d'inoculum $(\mathrm{v} / \mathrm{v})$ et 200 grammes de soutre élémentaire en granules dans le second, le premier servant de contrôle. Afin d'éviter des volumes morts et l'apparition de zones d'anaérobiose dans les réacteurs, ceux-ci sont aérés par le bas, et agités mécaniquement à l'aide d'un agitateur à hélice à 2 pales en plastique, à $80 \mathrm{tr} / \mathrm{min}$.

\section{Bioréacteur avec recirculation des boues de type colonne (R3)}

Dans ce cas, 3400 grammes de soufre en blocs sont introduits au fond de la colonne, avec 7 litres de boues à traiter et 1 litre d'inoculum. La recirculation des boues se fait par apport d'air à partir du bas de la colonne, ce qui minimise des volumes morts dans celle-ci.

\section{Essais de biolixiviation - stabilisation}

Pour réaliser ces essais, le soufre et l'inoculum étaient ajoutés aux boues, dans les bioréacteurs $R 2$ et $R 3$; le premier réacteur (R1) servant de contrôle ne contenait que les boues à stabiliser, n'ayant reçu ni soufre ni inoculum. Le taux d'aération pour chacun des bioréacteurs était égal à 0,3 volume/ volume/minute (v.v.m.). Les conditions expérimentales sont résumées dans le tableau 2 : 
Tableau 2 Conditions expérimentales des essais de biolixiviation-stabilisation.

Table 2 Experimental conditions for the bioleaching-stabilization experiments.

\begin{tabular}{|c|c|c|c|}
\hline & R1 & $\mathrm{R} 2$ & R3 \\
\hline Volume de boues (L) & 30 & 24 & 7 \\
\hline Inoculum (L) & 0 & 6 & 1 \\
\hline Température $\left({ }^{\circ} \mathrm{C}\right)$ & $20-22$ & $20-22$ & $20-22$ \\
\hline Aération (wm) & 0,3 & 0,3 & 0,3 \\
\hline Agitation (t $t / \mathrm{min})$ & 80 & 80 & par aération \\
\hline$[S . T]_{\text {initiale }}(g / l)$ & 20 & 24 & 21 \\
\hline$[S . V .]_{\text {initiale }}(g / l)$ & 13 & 15 & 12 \\
\hline Soufre ajouté (g) & 0 & 200 (granules) & 3400 (blocs) \\
\hline
\end{tabular}

\section{Pouvoir acidophile de la boue traitée}

Après chaque expérimentation, $200 \mathrm{~mL}$ de boue lixiviée étaient récupérés dans des Erlenmeyers, leur pH était réajusté à 7.0 avec de la soude $(\mathrm{NaOH})$, et le mélange était incubé à température ambiante dans un agitateur giratoire ajusté à $200 \mathrm{tr} / \mathrm{min}$. Le $\mathrm{pH}$ et le potentiel d'oxydo-réduction étaient alors mesurés journellement afin de suivre l'acidification du milieu et donc de vérifier s'il restait encore dans cette boue déjà lixiviée du soufre résiduel non oxydé et susceptible de l'être.

\section{Paramètres mesurés}

Les paramètres mesurés lors de nos essais étaient les suivants: $p H$, potentiel d'oxydoréduction (POR), métaux lourds dissous $(\mathrm{Cr}, \mathrm{Cu}, \mathrm{Fe}, \mathrm{Pb}, \mathrm{Zn}$ ), phosphore (P), solides totaux (ST), solides totaux volatiles (SV), matières en suspension (MES), matières volatiles en suspension (MVS), soufre soluble (sulfates).

\section{Mesures}

\section{Mesure de l'oxydation du soufre élémentaire}

La concentration de sulfates produits dans la boue, suite à l'oxydation du soufre élémentaire, était mesurée à tous les 48 heures par spectrophotométrie à émission atomique avec atomisation de l'échantillon dans un plasma d'argon (ICP-AES), modèle Atom Scan 25 de Thermo Jarrell Ash Corporation. Le $\mathrm{pH}$ et le POR étaient également mesurés aux 24 heures avec un potentiomètre «Fisher Accumet " Model 805 MP, afin de suivre l'acidification du milieu (résultant de la production d'acide sulfurique), ainsi que ses conditions oxydantes.

\section{Mesure de la solubilisation des métaux}

Comme pour le soufre, les métaux en solution étaient mesurés à tous les deux jours selon les méthodes préconisées par l'APHA (1989) en faisant 
appel à la spectrophotométrie à émission atomique (ICP-AES). Les mesures étaient effectuées sur la fraction liquide des boues, obtenue par centrifugation à $12000 \mathrm{tr} / \mathrm{min}$ pendant 30 minutes; les surnageants ainsi obtenus étaient ensuite fitrés sur membranes en polycarbonate de $2 \mu \mathrm{m}$ de porosité. Les concentrations en métaux dans les boues étaient déterminées après digestion acide de celles-ci par $\mathrm{HNO}_{3}, \mathrm{HCl}, \mathrm{HF}$ et $\mathrm{H}_{2} \mathrm{O}_{2}$ à $30 \%$, selon le protocole utilisé par le Ministère de l'Environnement du Québec (MENVIQ. 89. 12/213Méthode 1.3). Pour contrôler la qualité de nos analyses, nous avons digéré et analysé des étalons certifiés avec chaque série d'analyses (étalons MESS-2 et PACS-1, échantillons de sédiments fournis par le Conseil national de recherches Canada, CNRC, Ottäwa, Ontario). Les valeurs ainsi obtenues pour le chrome, le cuivre, le fer, le plomb, le zinc, le soufre et le phosphore se trouvaient à l'intẻrieur des gammes certifiées.

\section{Mesure de la stabilisation des boues}

Cette mesure était effectuée à tous les 2 jours, par évaluation de la réduction de la biomasse. Les solides totaux (ST) et les solides totaux volatiles (SV) étaient déterminés sur des volumes de $50 \mathrm{~mL}$ de boues séchées respectivement à $105^{\circ} \mathrm{C}$ et à $550^{\circ} \mathrm{C}$. Par contre, la mesure des matières en suspension (MES), et celle des matières volatiles en suspension (MVS), étaient réalisées sur les culots obtenus après centrifugation de $30 \mathrm{~mL}$ de boues pendant 30 minutes, à $12000 \mathrm{tr} / \mathrm{min}$. Les culots étaient lavés à l'eau désionisée, puis recentrifugés, et finalement portés à $105^{\circ} \mathrm{C}$ pour la détermination des MES, puis à $550^{\circ} \mathrm{C}$ pour la mesure des MVS.

\section{RÉSULTATS ET DISCUSSION}

\section{Oxydation du soufre élémentaire}

La présence de soufre élémentaire et d'inoculum dans les bioréacteurs R2 et $\mathrm{R} 3$ a provoqué une baisse importante du $\mathrm{pH}$ au cours de la période d'incubation. Cette baisse de $\mathrm{pH}$ est étroitement liée aux quantités de substrat et d'inoculum présents dans le milieu. En effet, une étude que nous avons réalisée en Erlenmeyer, avec du soufre en granules, a montré qu'un minimum de soufre $(2 g / L)$ était nécessaire pour avoir une vitesse optimum d'acidification du milieu, mais que seulement $40 \%$ de ce soufre présent était oxydé (Benmoussa et al. 1994). Aussi, le pH a atteint une valeur de 2.1 après 9 jours d'incubation dans le bioréacteur R2 ; cette valeur de $\mathrm{pH}$ (2.1) était obtenue après seulement 6 jours d'incubation dans le bioréacteur R3 (fig. 1a). On observe aussi une production de sulfates directement liée à la baisse du pH (oxydation biologique du soufre élémentaire) (fig. 1b), ainsi qu'une augmenta tion du POR (fig. 1c). Cette augmentation du POR (initialement négatif probablement à cause de l'anoxie du milieu, et à la présence probable de sulfures) est due au phénomène d'aération (pendant les premiers jours). La valeur importante du POR obtenue en fin de lixiviation est très probablement 

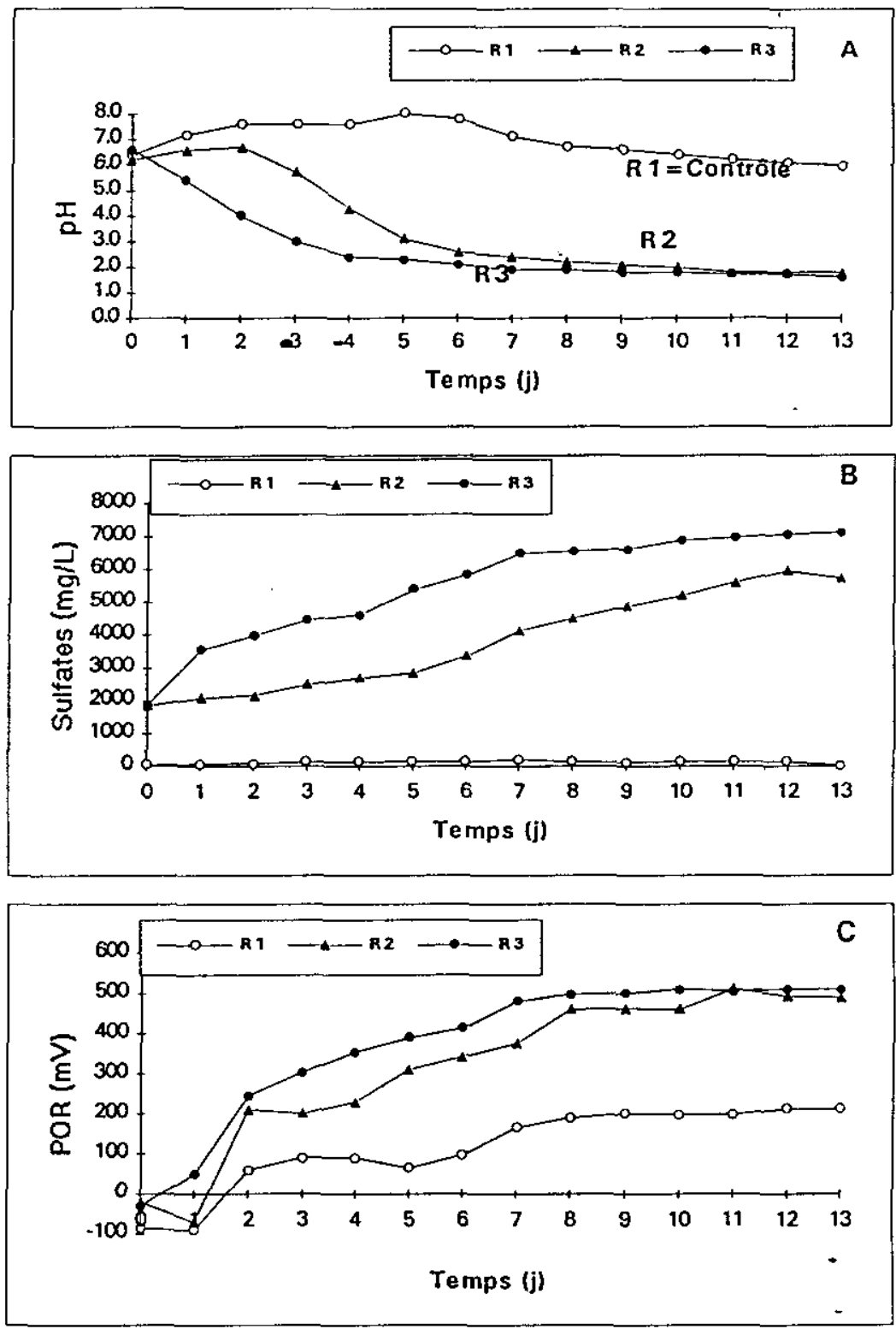

Figure 1 Variation du $\mathrm{pH}$, des sulfates et du potentiel d'oxydo-réduction au cours du temps.

- (R1) : pendant la stabilisation aérobie (contrôle).

- (R2) : pendant la biolixiviation-stabilisation en réacteur type RPA.

- (R3) : pendant la biolixiviation-stabilisation en colonne.

Variation of $\mathrm{pH}$, sulfates and oxido-reduction potential with time.

- (R1): during aerobic stabilization (control).

- (R2): during simultaneous leaching and stabilization in a stirred tank reactor (STR).

- (R3): during simultaneous leaching and stabilization in a column. 


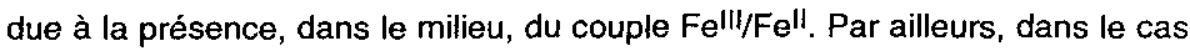
du bioréacteur $R 1$ (contrôle), nous observons d'abord une augmentation du $\mathrm{pH}$ (ammonification), suivie d'une faible acidification (fig. 1a) qui serait due au phénomène de nitrification (REYNOLDS, 1982 ; MARTIN, 1979) ; des travaux en cours de publication montrent, dans ce cas, une élimination de l'azote ammoniacal et une augmentation de la concentration en nitrates.

\section{Réduction des solides}

Les réductions des MES et des MVS sont présentées à la figure 2 ( $a$ et b), et sont respectivement de $54 \%$ et $50 \%$ pour le bioréacteur R3, et de $31 \%$ et $38 \%$ pour R1. Dans le cas du bioréacteur R2, ces réductions sont de $28 \%$ pour les MES, et de $32 \%$ pour les MVS. Les diminutions plutôt faibles dans R2 s'expliqueraient par la présence de petits granules de soufre résiduel non oxydé, qui ont été observés dans la boue de ce bioréacteur. Ces petits granules n'ont pu être séparés de la boue, et ont donc faussé nos mesures, ce qui explique les faibles taux de réduction de MES et de MVS dans ce bioréacteur (R2). II a aussi été observé qu'après 9 jours de biolixiviationstabilisation, ces valeurs demeuraient constantes pendant plusieurs jours, et les boues ainsi obtenues ont complètement perdu leur odeur initialement nauséabonde, ce qui démontre bien que la stabilisation était efficace.

Tout comme pour les MES et MVS, les concentrations en ST et en SV diminuent progressivement pendant les 10 premiers jours. Cette diminution était plus marquée dans les bioréacteurs $R 2$ et $R 3$ que dans le contrôle (bioréacteur R1) (tableau 3). Après 10 jours d'incubation, une légère augmentation de ces deux paramètres était notée. Ceci était dû à la production de sulfates au cours de la biolixiviation, et aussi à la présence de soufre élémentaire résiduel dans le cas du bioréacteur type RPA (R2). D'ailleurs cette augmentation n'était plus observée après soustraction des sulfates. Dans ce cas, les valeurs de pourcentage de réduction pour [ST $-\mathrm{SO}_{4}{ }^{2-}$ ] et pour [SV $-\mathrm{SO}_{4}{ }^{2-}$ ] sont respectivement de $56 \%$ et $51 \%$ pour le bioréacteur $\mathrm{R3}$, de $42 \%$ et $45 \%$ pour R2 et de $26 \%$ et $35 \%$ pour R1.

En effet, l'oxydation du soufre élémentaire donne lieu à une production d'acide sulfurique, volatile à $550^{\circ} \mathrm{C}$. Par ailleurs, la présence dans le milieu de l'ion ammonium $\left(\mathrm{NH}_{4}{ }^{+}\right)$, conduit à la formation de sulfate d'ammonium $\left(\left(\mathrm{NH}_{4}\right)_{2} \mathrm{SO}_{4}\right)$ (volatile à $\left.550^{\circ} \mathrm{C}\right)$, ainsi qu'à des sulfates mixtes renfermant à la fois l'ion ammonium et un ion métallique. Ces sulfates mixtes sont généralement partiellement volatiles (dissociation puis volatilisation de $\left(\mathrm{NH}_{4}\right)_{2} \mathrm{SO}_{4}$ ), ce qui se traduit par une augmentation des SV. Dans cette même optique, des essais réalisés avec des sulfates d'aluminium $\left(\mathrm{Al}_{2}\left(\mathrm{SO}_{4}\right)_{3}\left(\mathrm{NH}_{4}\right)_{2} \mathrm{SO}_{4}, 24 \mathrm{H}_{2} \mathrm{O}\right.$ et de fer $\left(\mathrm{Fe}\left(\mathrm{NH}_{4}\right)_{2}\left(\mathrm{SO}_{4}\right)_{2}, 6 \mathrm{H} 2 \mathrm{O}\right.$, montrent que, lorsqu'associés à l'ion $\mathrm{NH}_{4}{ }^{+}$, ces sulfates sont effectivement partiellement volatiles à $550^{\circ} \mathrm{C}$.

\section{Pouvoir acidophile de la boue déjà lixiviée}

A la fin de chaque série d'essais en bioréacteurs il est important de vérifier la présence de soufre résiduel non oxydé, et ce, en mesurant le pouvoir acidophile de chaque boue déjà lixiviée. Ces essais sont réalisés en Erlenmeyers 

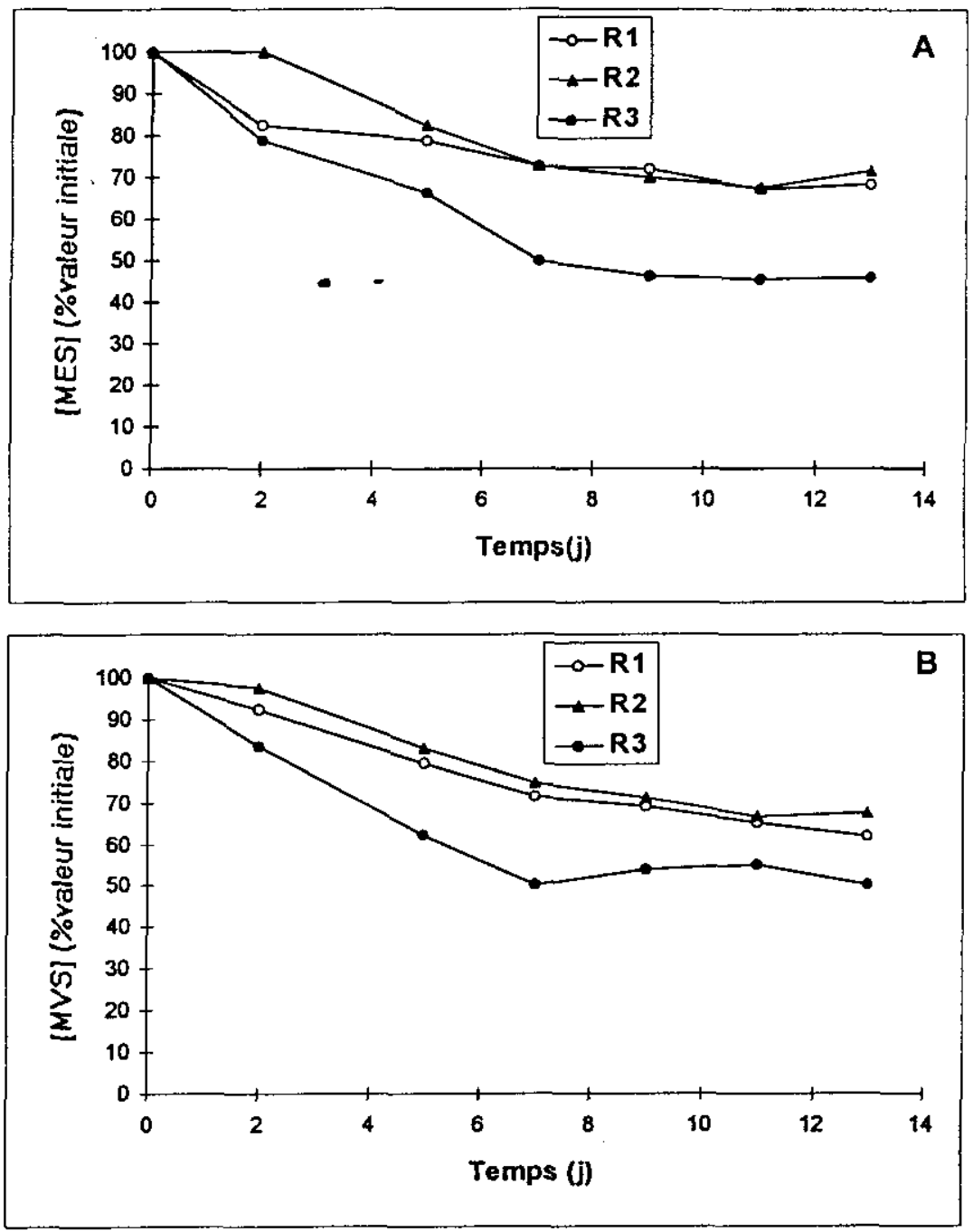

Figure 2 Réduction des MES et MVS au cours du temps.

- (R1) : pendant la stabilisation aérobie (contrôle).

- (R2) : pendant la biolixiviation-stabilisation en réacteur type RPA.

- (R3) : pendant la biolixiviation-stabilisation en colonne.

Reduction of suspended solids and volatile suspended solids with time.

- (R1): during aerobic stabilization (control).

- (R2): during simultaneous leaching and stabilization in a stirred tank reactor (STR).

- (R3): during simultaneous leaching and stabilization in a column. 

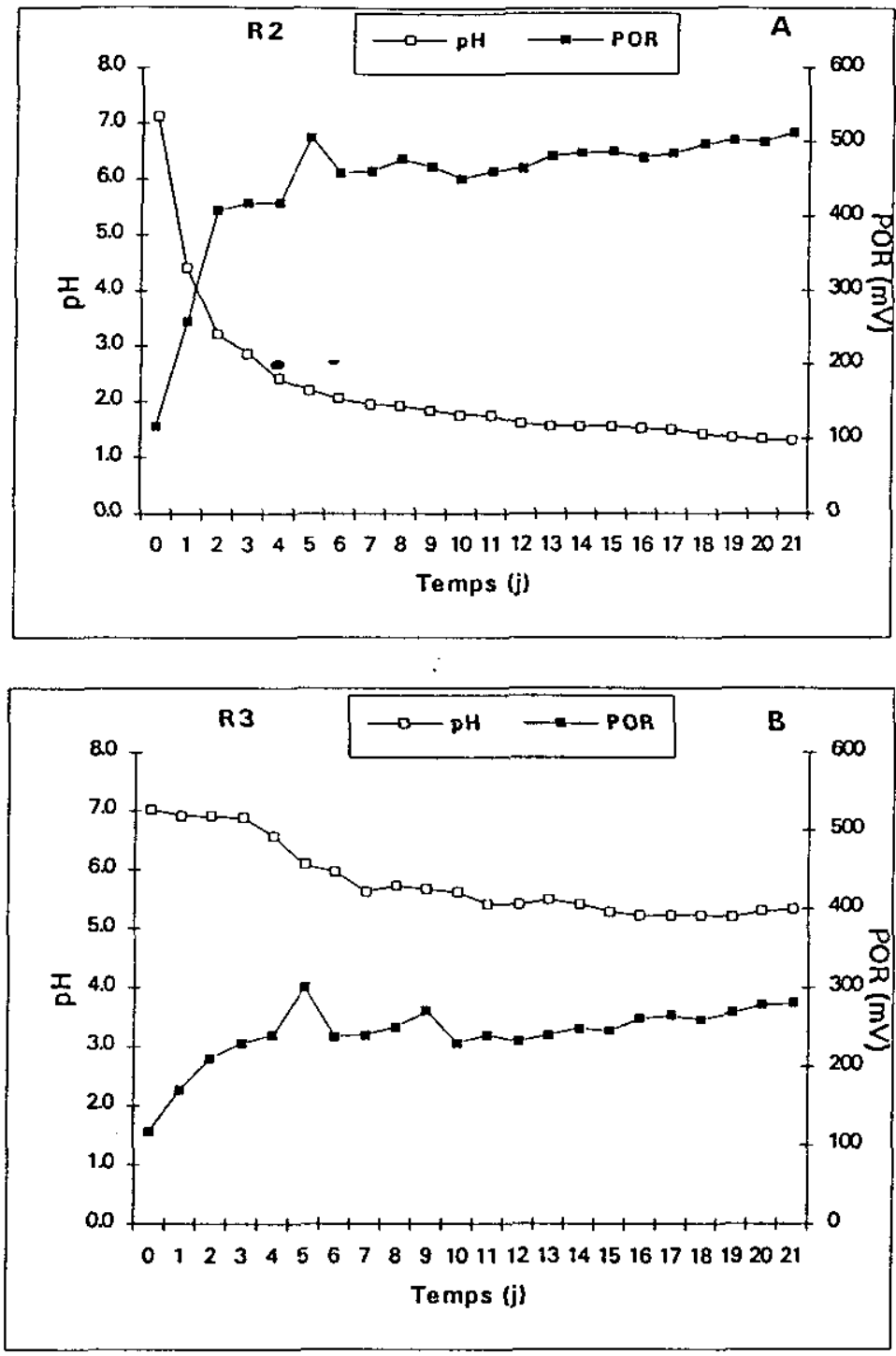

Figure 3 Vérification du pouvoir acidophile d'une boue déjà lixiviée en bioréacteur et ramenée à un $\mathrm{pH}$ de 7,0 . Variation du $\mathrm{pH}$ et du POR en fonction du temps.

- (R2) : boue provenant du bioréacteur type (RPA) ;

$\mathrm{pH}$ avant neutralisation $=1,80$.

- (R3) : boue provenant du bioréacteur type (colonne) ;

$\mathrm{pH}$ avant neutralisation $=1,61$.

Verification of the tendency of sludge leached in a bioreactor, and then neutralizd, to re-acidify. Variation of $\mathrm{pH}$ and $\mathrm{ORP}$ with time.

- (R2): sludge from stirred tank reactor (STR) ;

$\mathrm{pH}$ before neutralization $=1.80$.

- (R3): sludge from column type bioreactor;

$\mathrm{pH}$ before neutralization $=1.61$. 
de $500 \mathrm{ml}$, où $200 \mathrm{ml}$ de chaque boue déjà lixiviée en bioréacteur sont introduits, à $\mathrm{pH} 7$. Les résultats obtenus montrent une réacidification rapide pour les boues du réacteur R2 (fig. 3a), vraisemblablement due à la présence de soufre résiduel. La faible réacidification observée dans le cas des boues traitées dans le réacteur $\mathrm{R} 3$ serait plutôt due au phénomène de nitrification (REYNOLDS, 1982 ; MARTIN, 1979) (fig. 3b).

Tableau 3 Variation des solides après 13 jours :

- (R1) : pendant la stabilisation aérobie (contrôle).

- (R2) : pendant la biolixiviation-stabilisation en réacteur type (RPA).

- (R3) : pendant la biolixiviation-stabilisation en colonne.

Table 3 Variation of solids after 13 days:

$-(R 1)$ : during aerobic stabilization (control).

- (R2): during simultaneous leaching and stabilization in a stirred tank reactor (STR).

- (R3): during simultaneous leaching and stabilization in a column.

\begin{tabular}{|c|c|c|c|c|c|c|c|c|c|}
\hline & \multicolumn{3}{|c|}{ Réacteur R1 (contrôle) } & \multicolumn{3}{|c|}{ Réacteur R2 (type RPA) } & \multicolumn{3}{|c|}{ Réacteur R3 (colonne) } \\
\hline & Initial & Final & $\begin{array}{l}\% \text { de } \\
\text { réduction }\end{array}$ & Initial & Final & $\begin{array}{l}\% \text { de } \\
\text { réduction }\end{array}$ & initial & Final & $\begin{array}{l}\% \text { de } \\
\text { réduction }\end{array}$ \\
\hline [ST] $(g / L)$ & 20 & 15 & 26 & 24 & 19 & 22 & 22 & 16 & 27 \\
\hline$[S T](g / L)$ & 13 & 8 & 35 & 18 & 15 & 19 & 16 & 14 & 12 \\
\hline$[S T-S 04](g / L)$ & 20 & 15 & 26 & 22 & 13 & 42 & 20 & 9 & 56 \\
\hline$[S V-S 04](g / L)$ & 13 & 8 & 35 & 16 & 9 & 45 & 14 & 7 & 51 \\
\hline [MES] $(g / L)$ & 19 & 13 & 31 & 19 & 14 & 28 & 18 & 8 & 54 \\
\hline [MESV] $(g / L)$ & 13 & 8 & 38 & 14 & 9 & 32 & 12 & 6 & 50 \\
\hline
\end{tabular}

ST $=$ solides totaux $;$ SV $=$ solides totaux volatiles

MES = matières en suspension; MVS = matières volatiles en suspension.

\section{Solubilisation des métaux}

La forte chute de $\mathrm{pH}$ observée dans les réacteurs $\mathrm{R} 2$ et $\mathrm{R} 3$ entraîne une solubilisation importante des métaux. Les taux de solubilisation (exprimés en \%) des différents éléments variaient selon leur nature chimique de (Fe : 30 à $40 \%, \mathrm{P}: 52 \%, \mathrm{Cr}: 53$ à $56 \%, \mathrm{~Pb}: 69 \%, \mathrm{Cu}: 97 \%, \mathrm{Zn}: 98 \%)$, et les résultats obtenus sont représentés dans les figures 4 ( $a$ et b). Dans notre cas le taux de solubilisation (ou de mise en solution) des métaux, calculé à différents temps, représente le rapport de la concentration des métaux en solution sur la concentration des métaux contenus dans la boue initiale. Les concentrations en métaux résiduels dans les boues ainsi produites (calculées par différence entre les métaux contenus dans la boue initiale et ceux en phase liquide en fin d'expérience) respectent parfaitement les normes préconisées par le Gouvemement du Québec (1991) pour la disposition des boues en agriculture. Le faible taux de solubilisation du fer s'expliquerait par sa reprécipitation sous forme ferrique. It a d'ailleurs été remarqué que sa solubilisation évolue dans le même sens que celle du phosphore, et que lorsque l'aération est arrêtée, ces deux éléments repassent progressivement en solution. 

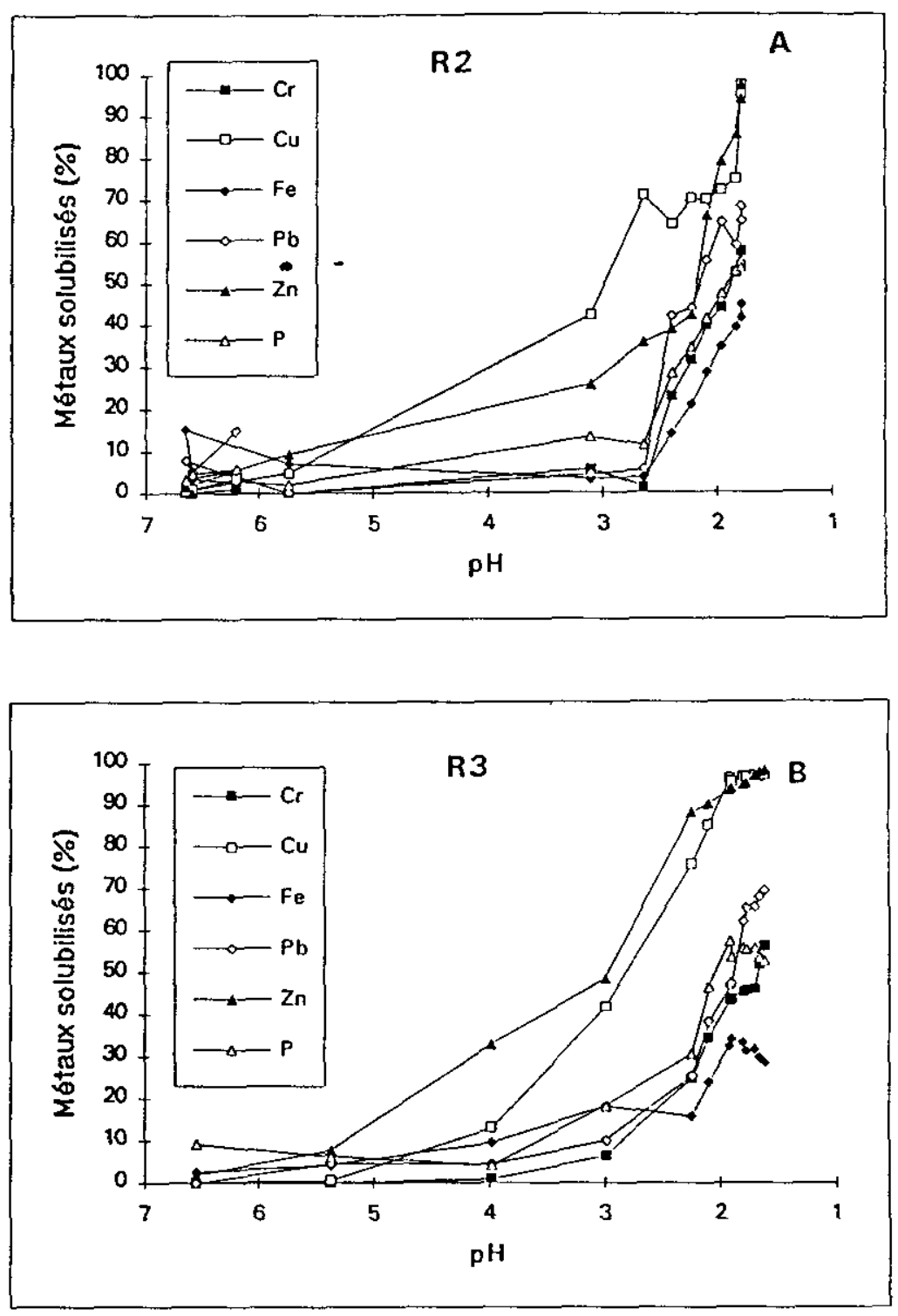

Figure 4 Solubilisation des métaux en fonction du $\mathrm{pH}$, pendant la biolixiviationstabilisation des boues dans les bioréacteurs R2 et R3.

Solubilization of metals as a function of $\mathrm{pH}$, during the simultaneous leaching and stabilization of sewage sludges in reactors $R 2$ and $R 3$. 


\section{CONCLUSIONS}

Les essais réalisés en réacteurs montrent qu'il est possible d'effectuer, à l'échelle du laboratoire, de la biolixiviation-stabilisation, en utilisant le soufre sous différentes formes: en granules (R2) ou en blocs dans le cas de la colonne (R3).

La fixation et la séparation du soufre élémentaire peuvent être aisément réalisées lorsque le soufre st utilisé sous forme de blocs, en colonne. Dans ce cas, le soufre peut être constamment réutilisé, et une acidification de la boue traitée n'est pas observée lorsque le $\mathrm{pH}$ de celle-ci est réajusté à 7 avec de la soude $(\mathrm{NaOH})$, ce qui laisse entendre l'absence totale de soufre élémentaire résiduel dans la boue, après lixiviation.

Le procédé biologique de solubilisation des métaux lourds examiné dans cette étude pourrait permettre la décontamination des boues d'épuration municipales à des niveaux compatibles avec l'utilisation de cette biomasse en agriculture.

La réduction importante des matières volatiles en suspension, observée lors de la biolixiviation-stabilisation, est très prometteuse eu égard à l'emploi de cette approche pour la stabilisation des boues d'épuration.

Enfin, cette biotechnologie pourrait s'avérer intéressante pour le traitement de diverses boues industrielles fortement contaminées en métaux toxiques.

\section{RÉFÉRENCES BIBLIOGRAPHIQUES}

APHA, 1989, Standards Methods for Examination of Water and Wastewaters. $17^{\circ}$ edition, APHA-AWWA-WPCF, Washington, D.C., U.S.A.

BENMOUSSA H., TYAGI R.D., CAMPBELL P.G.C., BLAIS J.F., 1994. Lixiviation biologique des métaux lourds et stabilisation des boues municipales. Wat. Pollut. Res. J. Can., 29, 1, 39-52.

BLAIS J.F., AUCLAIR J.C., TYAGI R.D., 1992a. Cooperation between two Thiobacillus strains for heavy metals removal from municipal sludge. Cal. J. Microbiol, 38 , 181-187.

BLAIS J.F., TYAGI R.D., AUCLAIR J.C., LAVOIE M.C.,1992b, Indicator bacteria reduction in sewage sludge by a metal bioleaching process. Wat. Res., 26, 487-495.
BRUCE A.M., DAVIS R.D.,1989. Sewage sludge disposal : current and future options. Wat. Sci. Technol, 21, 1113. 1128.

CHANEY R.L., 1990. Twenty years of land application research. Biocycle, septembre, 54-59.

COUILLARD D., MERCIER G, 1991a. Optimum residence time (in CSTR and airlift reactor) for bacterial leaching of metals from anaerobic sewage sludge. Wat. Res., 25 , 21 1-218.

COUILLARD D., MERCIER G, 1991b. Procédé de solubilisation biologique des métaux dans les boues anaérobies d'épuration : filtrabilité, neutralisation et teneurs en $\mathrm{N}$ et $\mathrm{P}$ des boues traitées. Can. J. Chem. Eng., $69,779-787$. 
DAVIS R.D., 1987. Use of sewage sludge on land in the United Kingdom. Wat. Sci. Technol., 19, 1-8.

DEGRÉMONT, 1989. Mémento Technique de l'eau. Neuvième édition, DEGREMONT, Paris, tomes 1 et 2.

GOUVERNEMENT DU QUÉBEC, 1991. Valorisation agricole des boues de stations d'épuration des eaux usées municipales. Guides de bonnes pratiques. Québec, Canada, Ministère de l'Environnement, Ministère de I'Agriculture des"Pêcheries et de I'Alimentation. ISBN 2-550-26359-6, Envirodoq EN920246, XVI +91p.

HUTCHINS S.R., DAVIDSON M.S., BRIERLEY J.A., BRIERLEY C.L.,1986. Microorganisms in reclamation of metals. Ann. Rev. Microbiol., 40, $311-336$.

JAIN D.K., TYAGI R.D., 1992. Leaching of heavy metals from anaerobic sewage sludge by sulfur-oxidizing bacteria. Enzyme Microb. Technol., 14,376-383.

LAISHLEY E.J., BRYANT R.D., KOBRYN B.W., HYNE J.B., 1986. Microcrystalline structure and surface area of elemental sulphur as factors influencing its oxidation by Thiobacillus albertis . Can. J. Microbiol. 32, 237-242.

LESTER J.N., STERRITT R.M., KIRK P.W.W., 1983. Significance and behaviour of metals in waste water treatment processes. I. Sewage treatment and effluent discharge. Sci. Total Environ. 30, 1-44.

MARTIN G., 1979. Le probleme de lazote dans les eaux, Technique et Documentation, Paris, $279 \mathrm{p}$.

MININNI G., SANTORI M., 1987. Problems and perspectives of sludge utilization in agriculture. Ecosystem Environ. 18, 291-311.

REYNOLDS T.D., 1982. Unit operations and processes in environmental engineering.
Ray Kingman (Editeur), 8/C Engineering Division, PWS Publishers, Boston, MA, U.S.A., $576 \mathrm{p}$.

TYAGI R.D., COUILLARD D., TRAN F.T., 1990. Studies on microbial leaching of heavy metals from municipal sludge. Wat. Sci. Technol. 22, 229-238.

TYLER G., BALSBERG PAKLSSON A.M., BENGTSSON G., BAATH E., TRANVUK L., 1989. Heavy metal ecology of terrestrial plants, microorganisms and invertebrates. A review. Wat. Air. Soil Pollut. 47, 189-215.

U.S. EPA, 1979. Sludge treatment and disposal. Process design manual. Center for Environmental Research Information, Cincinnati, U.S.A., EPA-625/1-79-011, 952 p.

U.S. EPA, 1990. National sewage sludge survey; availability of information and data, and anticipated impacts on proposed regulations, proposed rule. U.S. Fed. Register, 55, 47209-47283.

WEBBER M.D., 1988. Contrôle de la concentration de métaux lourds dans les sols après épandage de boues d'égout municipales : fapproche canadienne. Sci. Tech. Eau 21, 45-51.

WONG L., HENRY J.G., 1983. Bacterial leaching of heavy metals from anaerobically digested sewage sludge. Wat. Pollut. Res. J. Canada. 18, 151-162.

WONG L., HENRY J.G., 1988. Bacterial leaching of heavy metals from anaerobically digested sludge. Dans : Biotreatment Systems Vol. 2, D.L. Wise (éd.), CAC Press Inc., Boca Raton, Florida, U.S.A, 125-169.

WOZNIAK D.J., J.Y.C. HUANG J.Y.C., 1982. Variables affecting metal removal from sludge. J. Water. Pollut. Control Fed., 54, 1574-1580. 